\title{
INTEGRATED PERISTALTIC 18-STAGE ELECTROSTATIC GAS MICRO PUMP WITH ACTIVE MICROVALVES
}

\author{
Hanseup Kim, Aaron A. Astle, Khalil Najafi, Luis P. Bernal, and Peter D. Washabaugh \\ Center for Wireless Integrated MicroSystems (WIMS) \\ University of Michigan, Ann Arbor, MI 48109-2122, USA
}

\begin{abstract}
We report the design, fabrication, and testing of a fully integrated peristaltic 18-stage electrostatic gas micro pump with active microvalves. It consists of nine 2-stage pump units and 19 microvalves that are serially-connected and individuallycontrolled. It utilizes a number of techniques to achieve highpressure and high flow-rate performance: 1) multi-stage (18stage) configuration to distribute the total pressure across individual stages thus allowing each stage to operate at low pressure to accommodate the weak forces available in the micro scale; 2) a fluidic resonance-based operation to achieve high mass flow rate despite the small volume displacement of an individual membrane; 3 ) active timing control of microvalves to regulate pump operation for either high flow or high pressure; and 4) several new designs, such as checkerboard microvalves, dual-electrodes, and dual-chambers to achieve efficient electrostatic pumping. The fabricated 18-statge pump produced the highest air flow rate of $\sim 4.0 \mathrm{sccm}$ and maximum pressure difference of $\sim 17.5 \mathrm{kPa}$ with a total power of only $\sim 57 \mathrm{~mW}$. It has a total package volume of $25.1 \times 19.1 \times 1 \mathrm{~mm}^{3}$.
\end{abstract}

Key Words: Electrostatic pump, Micro gas pump, Peristlatic pump, Active microvalves.

\section{INTRODUCTION}

Recent literature has increasingly reported the importance of miniaturizing conventional 'bulk' chemical analysis or monitoring instruments in reducing analysis time, amount of target samples, cost, as well as in increasing analysis resolution, and portability. The miniaturization efforts cover numerous fields from environmental and health monitoring to bio- and pharmaceutical research, such as gas or water monitoring, blood or saliva analysis, cell or tissue handling, lab-on-a-chip, drug delivery, and drug tests. In spite of active miniaturization efforts and their recent success in sensing technology in the micro domain, micro-analysis devices still depend on conventional 'meso-scale' fluid delivery systems, such as large gas tanks or syringe pumps, to obtain high pressures and high flow rates [1]. The dependence on such 'bulky' fluidic systems prevents both a true miniaturization of the systems and precise control of small doses of chemicals and bio-subjects.

Similar challenges exist to the development of a micro gas chromatography $(\mu \mathrm{GC})$ system under development at the Center for Wireless Integrated MicroSystems (WIMS) at the University of Michigan [2]. The WIMS $\mu \mathrm{GC}$ is a highly complex microsystem and requires high-flow rate and a high-pressure gas flow to support the operation of other micro components such as a pre-concentrator, micro columns, and micro sensors. Previous gas micropumps have shown only limited capabilities, such as low flow-rate, low pressure, and large volume, thus failing to meet the requirements of the WIMS $\mu \mathrm{GC}$. Generally, flow rate is limited by the small deflections of a pumping membrane, while pressure is limited by the small actuation forces available in the micro domain. Additionally, micro pumping becomes unpredictable due to gas compressibility above a certain frequency.

Theoretical studies have shown that a high flow rate, high pressure, low power, and small gas micro pump could be realized by 'pressure accumulation' through a low-compression multi-stage configuration and 'high-speed volume displacement' through a highfrequency resonant operation of a pumping membrane in an electrostatic micro pump [3].

In this paper, we report the fully integrated peristaltic 18 -stage electrostatic gas micro pump with active microvalves that achieves high-pressure and high flow rates by such 'pressure-accumulation' and 'high-speed volume displacement'. We also report the operation of checkerboard-shape microvalves and their influences on pumping.

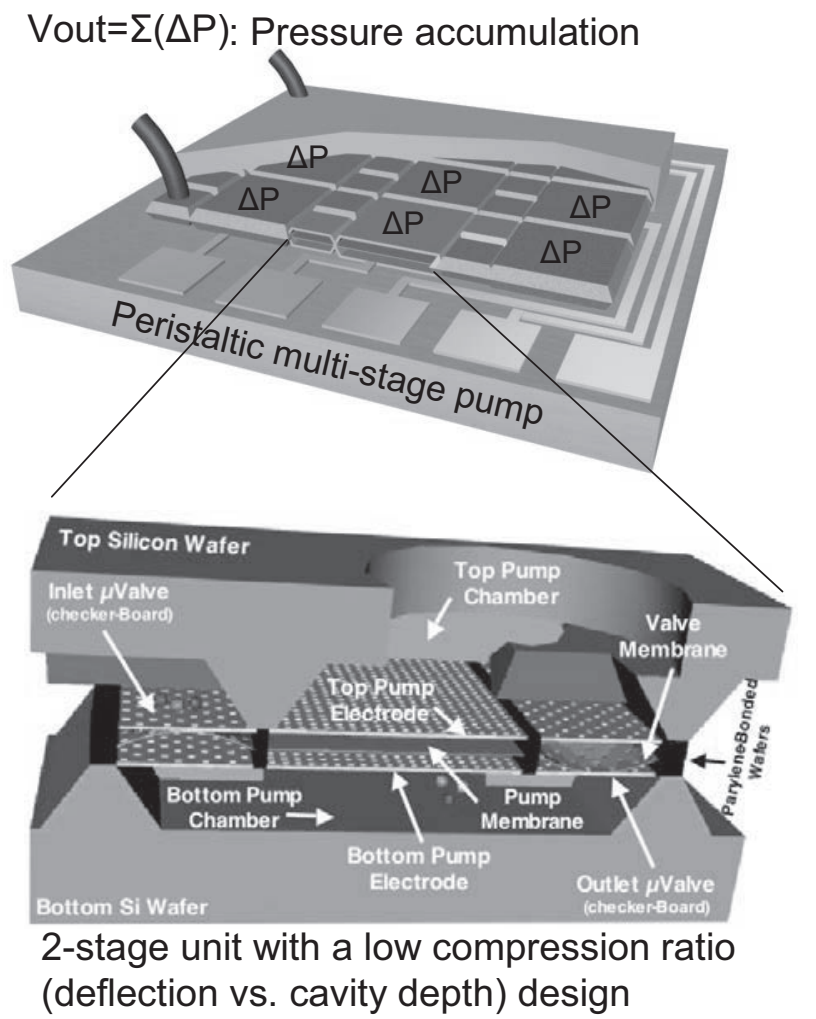

Figure 1: Illustration of (top) an integrated multi-stage (18-stage) micropump for high-pressure generation and (bottom) the detailed structure of a single pump unit (2-stage) formed by two bondedwafers. 

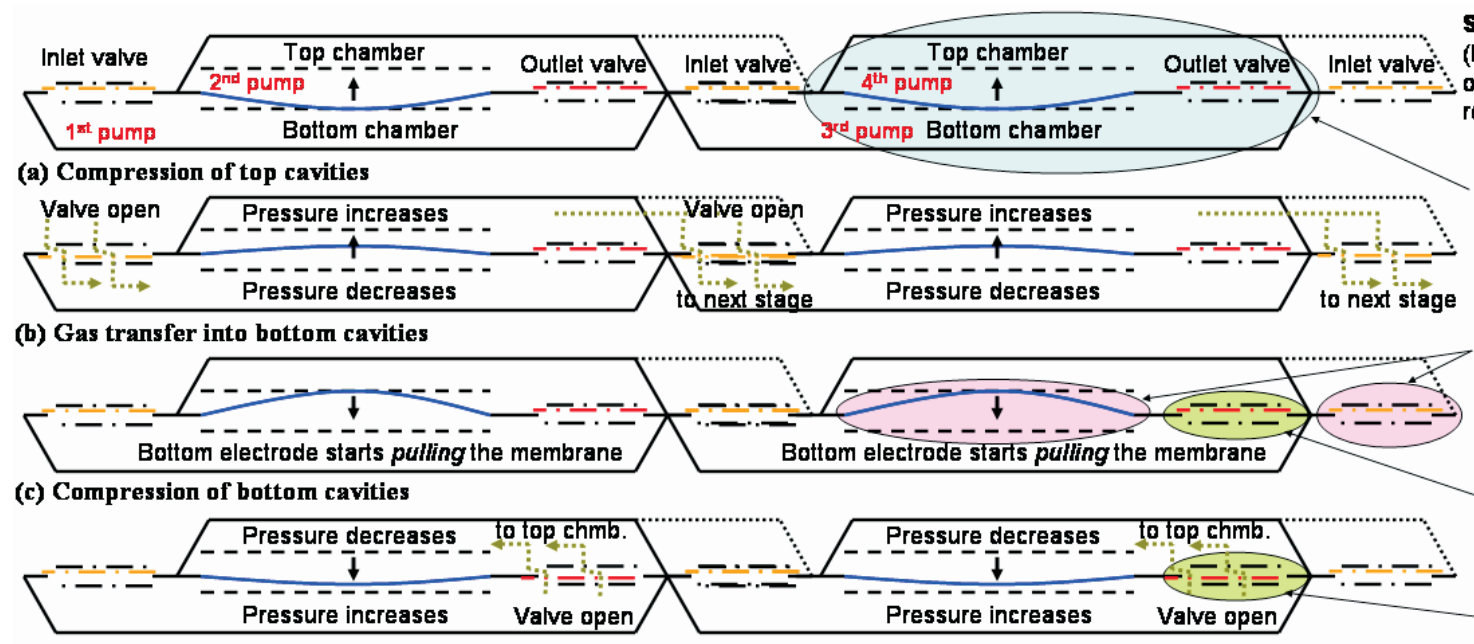

Synchronized motions of pump (blue), inlet valve (orange), and outlet valve (red) membranes. respectively.

Dual chamber (pump) structure sharing a single membrane $\rightarrow$ reduction in pump areas.

Dual electrode operation for bi-directional membrane movements $\rightarrow$ Increase stroke volumel microvalve control

Microvalves become closed when membrane holes do not match those of electrodes. (See fig. 4 also) Microvalves become open when membrane holes match (d) Gas transfer into top cavities

Figure 2: Operation principle of the reported multi-stage micropump. Respectively synchronized membrane movements generate peristaltic air-flows to adjacent pump stages. Air-flows come into the $1^{\text {st }}$ pump chamber at bottom during a gas transfer period (b), then move out into the $2^{\text {nd }}$ pump chamber on top during the next gas transfer period (d). This process repeats to next-stages.

\section{OPERATION}

The 18-stage pump distributes the overall pressure uniformly across individual stages and accumulates it for a high pressure difference across the whole pump (Figure 1-(top)). It is notable that such pressure-accumulation becomes possible through a low-compression design, where the volume compressed by a membrane is small compared to the overall pumping chamber volume [3]. In contrast, a simple cascade of several micro pumps generally leads to performance degradation due to non-uniform pressure distribution and thus pressure overloading in the last few stages [4, 5].

The 18-stage pump utilizes pumping membranes that are actuated at a high-frequency fluidic resonance and generates a high mass flow rate. The pumping membranes are made from flexible polymer films and are electrostatically-actuated by dual electrodes in two directions. The use of 'large-deflection' flexible polymer and 'pull-pull' dual-electrodes maximizes the gas flow rate.

The 18-stage pump consists of nine serially-connected 2stage units (Figure 1-(bottom)). Each 2-stage unit includes two (dual) pumping chambers formed in the top and bottom wafers and inlet and outlet (transfer) microvalves formed between the two bonded-wafers. Dual-pumping chambers reduce the overall size of the pump by stacking two chambers on top of each other and sharing a single pumping membrane. Inlet and outlet microvalves consist of one polymer valve membrane and two silicon electrodes, all of which contain checkerboard-type array of holes. The microvalve opens or closes gas flow depending on the match or mismatch of holes between the valve membrane and one of the dual electrodes.

Figure 2 shows the peristaltic operation of the 18-stage micro pump. In this peristaltic pump, gases progress according to pressure changes in adjacent pumping chambers. As the main pumping membrane moves up and expands the bottom chamber with all the microvalves closed, the pressure in the first (bottom) chamber becomes lower (Figure 2 (a)). When the pressure in the first (bottom) chamber becomes low enough to introduce atmospheric gases into the chamber, the inlet "checkerboard" microvalve opens by a separate control signal and gases are introduced into the micro pump (Figure 2 (b)). When the pumping membranes reach the top, all the valves are closed and the next cycle begins. In the next cycle, the pressure in the first (bottom) chamber increases, as the main pumping membrane moves down and compresses the bottom chamber with all the microvalves closed (Figure 2 (c)). When the pressure in the first (bottom) chamber becomes higher than the adjacent second (top) chamber, the outlet valve opens and gases transfer from the bottom to the top chamber (Figure $2(\mathrm{~d})$ ). The same process repeats through the rest of the stages. Please note that as the pumping membrane moves down (up), it not only compresses (expands) the bottom chamber into high (low) pressure, but also expands (compresses) the top chamber into low (high) pressure simultaneously. Also please note that an outlet valve of the previous stage is an inlet valve of the next adjacent stage.

Figure 3 shows the timing control of integrated microvalves. The timing control over the valve opening duration determines the flow rate and pressure rise of the pump. When the opening duration is long (close to half the cycle: $0.5 \mathrm{~T}$ ), the pump flow rate is maximized and the pressure rise is very small because most of the gas just transfers because it was being confined and compressed. In this case the pump operates in the high-flow rate timing mode (HFT). On the other hand, when the opening duration is short $(0.25 \mathrm{~T})$, the duration of the membrane compression is increased and, therefore, the pressure increases and the flow rate decreases, which is defined as the high-pressure timing mode (HPT).

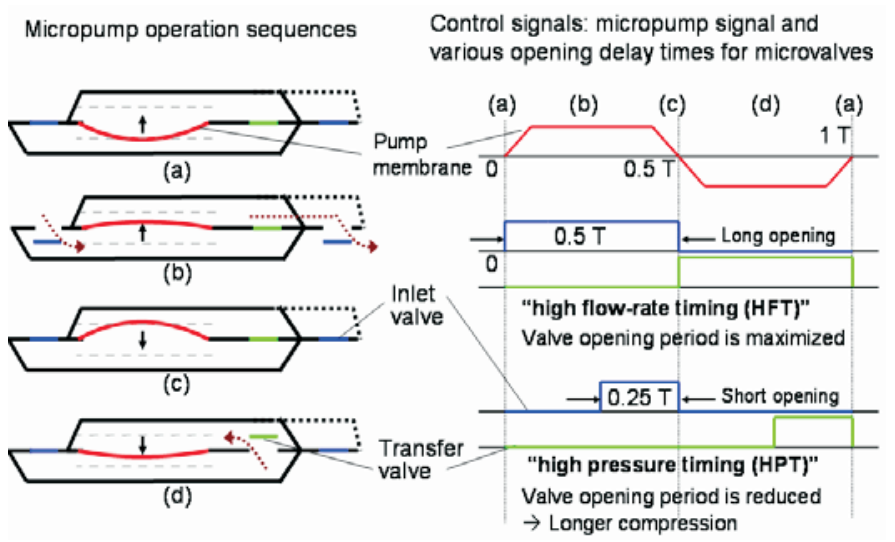

Figure 3: Microvalve timing control: HFT for high flow rate and HPT for high pressure pumping. 


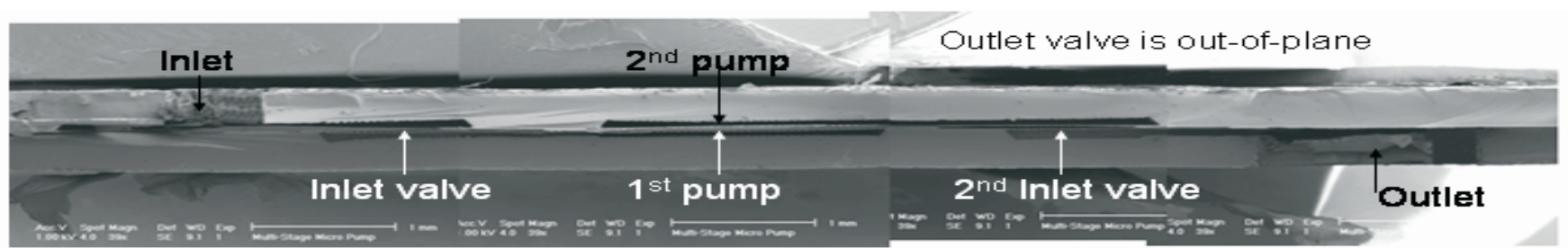

Figure 5: SEM of the cross-section of a fabricated two-stage micropump. A multi-stage micropump simply has a repeated form of such a structure.

\section{FABRICATION}

Figure 4 describes the microfabrication process for the multi-stage micro pump [6]. Fabrication consists of wafer bonding and membrane transfer among three wafers. First, a Parylene membrane is transferred from a carrier wafer onto a device wafer that contains pumping chambers, fluidic channels, and electrostatic electrodes. The transfer is performed at a wafer-level and constructs a thin $(\sim 1.5 \mu \mathrm{m}$ thick $)$ membrane that is suspended over the perforated electrode $(\sim 2000 \mu \mathrm{m})$ by a small gap $(\sim 5 \mu \mathrm{m})$ [7]. Then, a second device wafer is bonded to the first at a low temperature $\left(\sim 230^{\circ} \mathrm{C}\right)$ [8], thus sandwiching the pump and microvalve membranes between two electrodes and closing off the pump chamber.

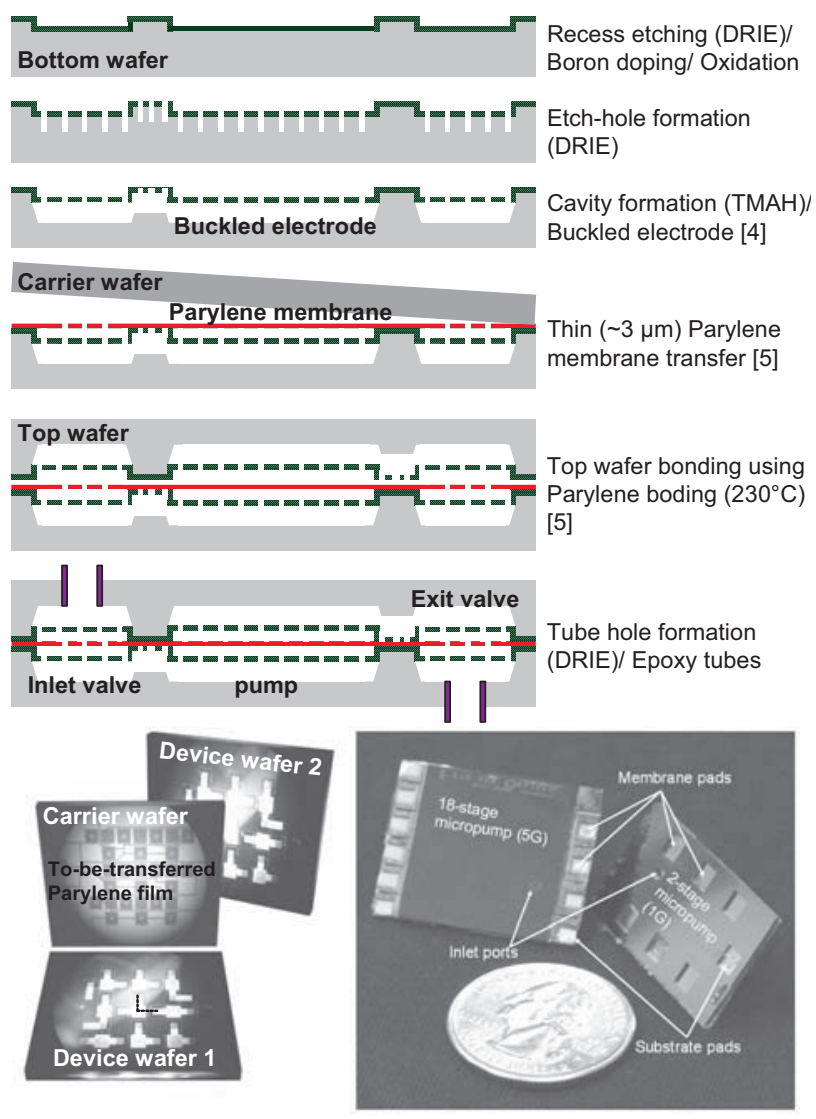

Figure 4: Microfabrication process flow [6].

\section{TEST RESULTS AND DISCUSSION}

The fabricated 18-stage micro pump as well as 4-stage and 2-stage pumps were operated at different voltages, frequencies, and microvalve timing, while being monitored using electronic mass flow meters and pressure transducers. First, the 18-stage pump is operated at different frequencies using a square wave voltage signal of $\pm 100 \mathrm{~V}$ to determine fluidic resonant frequency where highest flow rate or high pressure is generated. This test was performed under high flow timing (HFT) and high pressure timing (HPT), respectively. Then, at each resonance frequency, the fabricated micro pumps were operated under different load conditions to obtain the flow-vs.-pressure performance plot. Finally, they were used to pump air to move liquid droplets and columns in order to visualize and quantify performance.

\section{Flow rate vs. operation frequency}

Fabricated 18-, 4-, and 2-stage micropumps generated highest air flow rates of $\sim 4.0,3.0$, and $2.1 \mathrm{sccm}$ under the high flow rate timing (HFT), as shown in Figure 6. They consumed 57.0, 15.1, and $9.1 \mathrm{~mW}$. The 18-stage micropump has a resonance frequency at 17 $\mathrm{kHz}$, while 4-stage and 2-stage pumps have resonance peaks at 14 $\mathrm{kHz}$. Note that these measured flow rates are achieved by actuating membranes that are only $2 \times 2 \mathrm{~mm}^{2}$. When a pumping efficiency is defined by the flow rate to the membrane stroke volume, this result shows the highest efficiency by any membrane-based MEMS micro pump.

\section{Pressure vs. operation frequency}

Fabricated 18-, 4-, and 2-stage micro pumps produced maximum pressure differences of $\sim 17.5,7.0$, and $2.5 \mathrm{kPa}$ under the high pressure timing (HPT), as shown in Figure 7. These maximum pressure differences were obtained at an operation frequency of $\sim 15$ $\mathrm{kHz}$. Please note that the maximum pressure differences were obtained at different operation frequencies compared to the highest flow rates. The maximum pressure difference increases as the number of stages in the micro pump increases. The measured maximum pressure of $17.5 \mathrm{kPa}$ is the highest pressure generated by any electrostatic MEMS micro gas pump. Additionally, such pressure-accumulation through the multiple stages is realized for the first time by any MEMS pump.

\section{Pressure vs. flow rate for different microvalve timing}

Fabricated 18-, 4-, and 2-stage micro pumps demonstrated (1) that the micro pump has multiple performance lines (HFT and HPT) depending on the microvalve timing and (2) that the micro pump performance is easily controlled by varying the microvalve timing, and (3) that a higher pressure is achieved by using HPT, and a higher flow rate is achieved by using HFT, as shown in Figure 8. As shown, each fabricated micro pump has at least two different performance lines (HFT and HPT). For example, an 18-stage micro pump generates a higher maximum pressure $(17.5 \mathrm{kPa})$ using HPT than using HFT $(12.8 \mathrm{kPa})$. Similarly, the pump produces a higher maximum flow rate $(4.0 \mathrm{sccm})$ using HFT than using HPT $(2.6 \mathrm{sccm})$.

\section{Visualization}

Finally, the fabricated micro pumps were operated to visualize the gas pumping capability by pumping liquid droplets from one end to the other end of a tube. Figure 9 shows a sequence of photos illustrating the movement of a liquid droplet by air being pumped behind the droplet by a 4-stage pump. The droplet is ethanol, and the tube has a diameter of $1.56 \mathrm{~mm}$. 


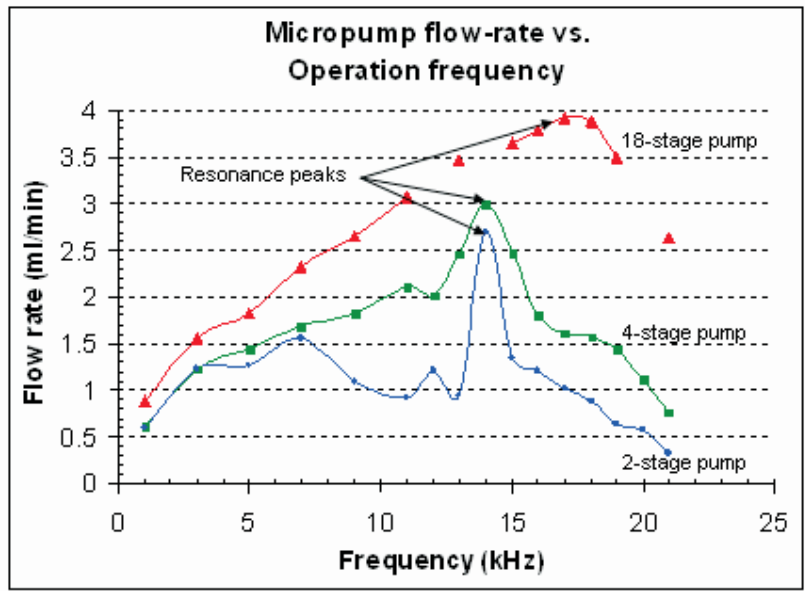

Figure 6: Mass (gas) flow rate result measured using electronic flow meters (Omega FM1600 series) over a wide range of frequencies. An 18-stage micropump has a resonance frequency at $17 \mathrm{kHz}$, while 4-stage and 2-stage pumps have resonant peaks at $14 \mathrm{kHz}$.

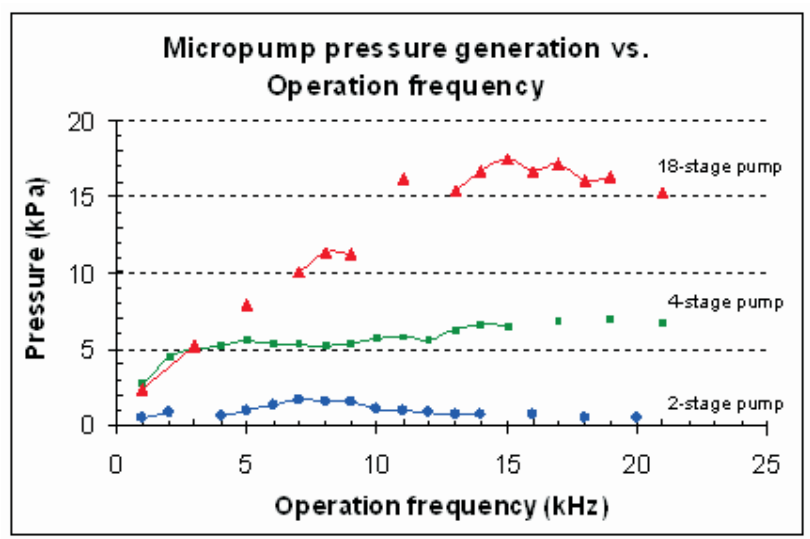

Figure 7: Measured pressure difference over a wide range of operation frequency. The pressure was measured using electronic pressure transducers (Validyne DP-45).

\section{CONCLUSIONS}

We have developed fully integrated 18-, 4-, and 2-stage peristaltic electrostatic micro gas pumps with active microvalves. The microfabricated pumps 1) achieve high pressure $(17.5 \mathrm{kPa})$ by accumulating pressure differences across multiple stages, 2$)$ obtain high flow rate $(4.0 \mathrm{sccm})$ by operating at a high fluidic resonance frequency $(\sim 15 \mathrm{kHz})$ despite a small membrane size $\left(2 \times 2 \mathrm{~mm}^{2}\right)$, while consuming only small power ( $\sim 57 \mathrm{~mW}), 3)$ have demonstrated pumping control (high flow rate or high pressure generation) by adjusting the microvalve opening duration (HFT or HPT); and 4) successfully implemented new designs, such as checkerboard microvalves, dual-electrodes, and dual-chambers to achieve efficient electrostatic pumping.

\section{ACKNOWLEDGEMENT}

This project is funded by the Engineering Research Centers Program of the National Science Foundation under Award Number EEC-9986866.

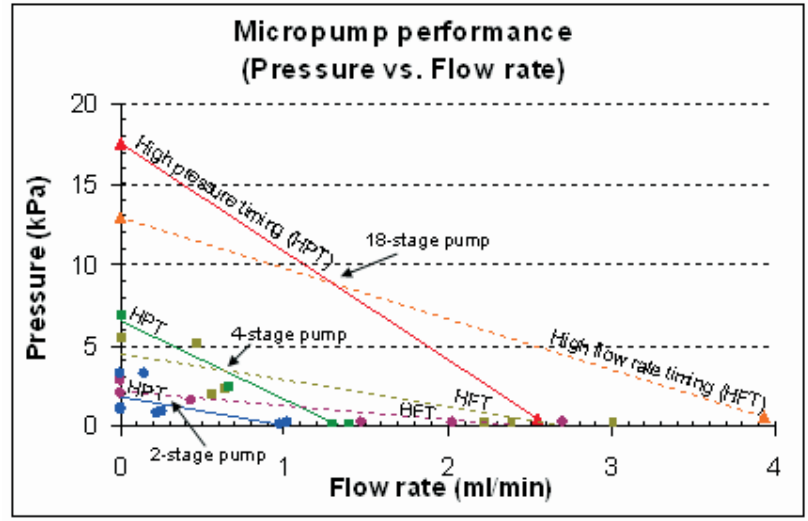

Figure 8: Pressure versus flow rate measurement results of different number of stage micropumps.
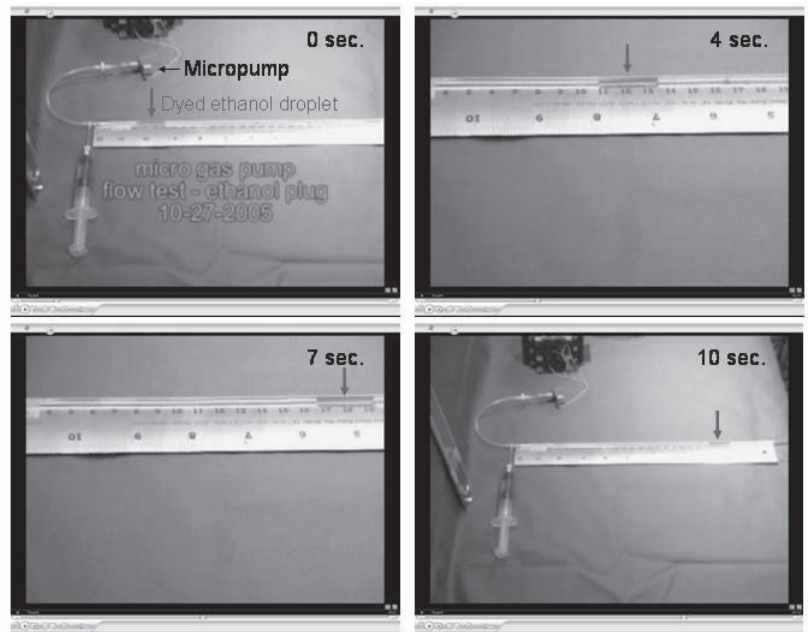

Figure 9: Visualization: ethanol drop is pumped through a thin tube (flow rate: $1.1 \mathrm{sccm}$ ) using air being pumped by a gas micro pump.

\section{REFERENCES}

[1] G. C. Frye-Mason et. al, "Microfabricated gas phase chemical analysis systems," Microprocesses and Nanotechnology Conference, pp. 60-61, 1999.

[2] E. Zellers et.al., "A versatile MEMS gas chromatograph for determinations of environmental vapor mixtures," Hilton Head '04, Hilton Head Island, SC, pp. 61-66, 2004.

[3] A. Astle, A. Paige, L. P. Bernal, J. Munfakh, H. Kim, and K. Najafi, "Analysis and design of multistage electrostaticallyactuated micro vacuum pumps," ASME IMECE, 2002-39308.

[4] R. Zengerle, A. Richter, and H. Sandmaier, "A micro membrane pump with electrostatic actuation," MEMS '92, Travemunde, Germany, pp. 19-24, 1992.

[5] M. Stehr, H. Gruhler, H. Straatmann, S. Messner, H. Sandmaier, and R. Zengerle, "The selfpriming VAMP," Transducers '97, Chicago, pp. 351-352, 1997.

[6] H. Kim, K. Najafi, A. Astle, L. Bernal, and P. Washabaugh, "Fabrication and performance of a dual-eledctrode electrostatic peristaltic gas micropump," $\mu$ TAS '05, pp. 1173-1176, pp. 2005.

[7] H. Kim and K. Najafi, "Wafer bonding using Parylene and waferlevel transfer of free-standing Parylene membranes," Transducers '03, Boston, MA, USA, pp. 790-793, 2003.

[8] H. Kim and K. Najafi, "Characterization of a low-temperature wafer bonding using thin film Parylene," IEEE Journal of Microelectromechanical Systems (JMEMS), vol. 14, no. 6, pp. 1347-1355, 2005. 